\title{
Pour une anthropologie plébéienne et pragmatiste du patrimoine
}

En finir avec le patrimoine patricien. Par le bas et par la marge

For a plebeian and pragmatist anthropology of the heritage, finishing off

patrician heritage, from below and from the margins

\section{Noël Barbe}

\section{OpenEdition}

\section{Journals}

Édition électronique

URL : https://journals.openedition.org/insituarss/485

DOI : 10.4000/insituarss.485

ISSN : 2680-4972

\section{Éditeur}

Ministère de la Culture

Référence électronique

Noël Barbe, « Pour une anthropologie plébéienne et pragmatiste du patrimoine », In Situ. Au regard des sciences sociales [En ligne], 1 | 2019, mis en ligne le 15 octobre 2019, consulté le 16 décembre 2022 URL : http://journals.openedition.org/insituarss/485; DOI : https://doi.org/10.4000/insituarss.485

Ce document a été généré automatiquement le 16 décembre 2022.

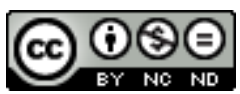

Creative Commons - Attribution - Pas d'Utilisation Commerciale - Pas de Modification 4.0 International - CC BY-NC-ND 4.0

https://creativecommons.org/licenses/by-nc-nd/4.0/ 


\title{
Pour une anthropologie plébéienne et pragmatiste du patrimoine
}

\author{
En finir avec le patrimoine patricien. Par le bas et par la marge \\ For a plebeian and pragmatist anthropology of the heritage, finishing off \\ patrician heritage, from below and from the margins
}

Noël Barbe

Pour U. M.

1 Il s'agira ici de faire travailler la question d'un patrimoine plébéien, en tant qu'il s'oppose à un patrimoine patricien et, pour cela, de déployer en regard certains des écarts et des oppositions mis à l'épreuve d'une pratique située et incarnée, c'est-à-dire effectuer un travail tout à la fois de recherche et d'action, en tant que ce lien peut s'établir et se faire varier, en somme être négocié dans la situation de conseiller pour l'ethnologie ${ }^{1}$, par conséquent dans une position embarquée ${ }^{2}$ dans une épistémologie politique de l'action patrimoniale - je reviendrai plus loin sur cette notion - et une politique de gestes, mais doté aussi d'une boussole politique réglée au fil du temps de l'exercice et des projets. Cette boussole, qui n'était pas donnée d'avance, se déploie tant biographiquement qu'intellectuellement, « depuis le bas » et « depuis la marge $»^{3}$.

2 La question appelle à tout le moins une précision d'abord, ensuite un retour - qui peutêtre n'en est d'ailleurs pas un. "Patrimoine plébéien» ne doit pas se comprendre comme ce qui viendrait catégoriser un ensemble de biens entretenant une relation particulière, faite d'historicité et d'attribution de valeurs, à une singularité sociale que serait la plèbe alors substantiellement saisie ${ }^{4}$. Ce qui ici se désigne est une modalité, plébéienne, de "faire patrimoine», disons de faire travailler l'historicité de biens auxquels une valeur dite patrimoniale est attribuée - et ce de différentes manières.

3 Un retour. Cette position s'inscrit dans une pratique des sciences sociales pensée comme une tentative, portée par des intérêts pratiques, d'élucidation du présent pris dans une historicité - comprendre les conditions d'une action dans l'histoire, avoir le souci de l'émancipation, comprise ici provisoirement comme la dilatation d'une 
capacité d'agir, ne pas se satisfaire de l'état du monde social ni être convaincu de sa nécessité -, pour reprendre la lecture que Jürgen Habermas (2005) fait de la naissance de la sociologie, analysant des auteurs écossais des Lumières. Produire des formes d'intelligibilité dans de telles coordonnées, c'est parfois se voir opposer des jugements de faits au nom de la fameuse "neutralité axiologique ", la Wertfreiheit wébérienne qu'Isabelle Kalinowski (2005) préfère traduire, rappelons-le, par «non-imposition des valeurs ». À ce propos, Philippe Chanial (2011: 34) pointe que Weber ne cesse de souligner que l'accès à ce qui est ne peut s'opérer qu'à travers un "rapport aux valeurs ». Et de citer les Essais sur la théorie de la science soulignant que ce qui est digne de connaissance, digne d'enquête pour le chercheur, c'est d'abord ce qui lui importe, ce qui fait sens et présente une certaine « valeur » à ses yeux : «Une portion seulement de la réalité singulière prend de l'intérêt et de la signification à nos yeux, parce que seule cette portion est en rapport avec les idées de valeurs culturelles avec lesquelles nous abordons la réalité concrète » (Weber $1992: 157)$.

\section{Épistémologie du positionnement et production de savoir}

4 Max Weber (1992: 160) indexe donc l'intérêt scientifique sur l'engagement: "Nous sommes des êtres civilisés, doués de la faculté et de la volonté de prendre consciemment position face au monde et de lui attribuer un sens.» Et Chanial (2011: 35) d'enchérir : «Cette faculté ne saurait être le monopole du savant. » Plus loin, Weber (1992: 161) écrit: «Il en résulte que toute connaissance de la réalité culturelle est toujours une connaissance à partir de points de vue spécifiquement particuliers. » Le cœur du positivisme repose sur le postulat d'une séparation du sujet et de l'objet ${ }^{5}$, et l'imputation aux conditions d'existence d'une "relation de connaissance troublée, obscurcie, voilée » (Foucault 2001: 1420). Renversant le propos, nous suivrons ici Michel Foucault lorsqu'il entend montrer que les « conditions politiques, économiques d'existence ne sont pas un voile ou un obstacle pour le sujet de connaissance, mais ce à travers quoi se forment les sujets de connaissance, et donc les relations de vérité " (ibid. : 1420-1421). Nous le suivrons loin de la neutralité axiologique ou du positivisme, au ras d'une forme négociée et revendiquée de l'exercice de conseiller pour l'ethnologie. Et ce via un détour épistémologique: l'installation et le réglage d'un dispositif de recherche pour une exposition sur Louis Pergaud, à la Ferme Courbet à Flagey (Doubs), nous en donnent l'occasion ${ }^{6}$. 


\section{Libraire:Bibliotherpua Anguste-Conte \\ 16, rue Saint-S6̈verin}

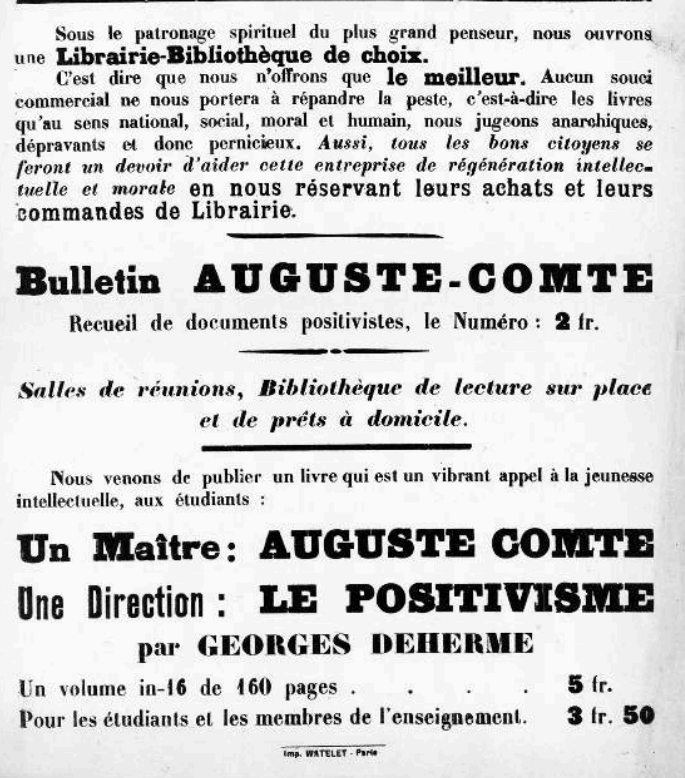

Le terme «positivisme » est attaché à Auguste Comte, acteur de l'histoire de la sociologie, qui invite à fonder une science d'abord appelée « physique sociale » puis nommée « sociologie » à partir de 1839. Comte théorise un régime social en relation avec un ordre industriel et un régime autoritaire et hiérarchique. Chacun y occupe une place dans l'interdépendance (la « solidarité ») générée par la division du travail social, qui se reproduit au fil du temps selon un processus de legs

intergénérationnel (la « continuité »). Le positivisme comtien exhorte au gouvernement par la science. C'est ainsi qu'Émile Littré, promoteur de Comte, fait de la sociologie l'un des fondements de la République conservatrice qui vient clore la Commune de Paris, et avec elle un siècle de révolutions. Avec l'apparition de cette nouvelle science, la politique se voit neutralisée techniquement et, pour reprendre l'expression de Jacques Donzelot, réduites les « passions politiques ».

(c) Photo G. Deherme, librairie-bibliothèque Auguste Comte / Maison d'Auguste Comte.

En 1915, l'auteur de La Guerre des boutons, originaire du premier plateau du département du Doubs, meurt à la guerre. En 2015, tout semble dit dans cette phrase pour les promoteurs des commémorations de sa mort qui entendent faire de l'écrivain un modèle d'obéissance civique et de discipline, parce que s'étant d'abord opposé puis rallié à la guerre, qui entendent minorer la portée politique de son œuvre ou de ses positions sur le mode d'un «ce n'est pas si grave ", s'appuyant sur l'une de ses œuvres ou plutôt sur l'un des films qui en a été adapté, i.e. La Guerre des boutons - assorti de la fameuse réplique, «Si j'aurais su, j'aurais pas venu ${ }^{7}$ "-, et enfin qui entendent le monétiser dans un essai de marketing territorial - sans même parler d'autres encore, désireux d'assigner Pergaud, en des accents quasi-pétainistes ou maurrassiens, à la place d'enfant du pays, au nom d'un : « Il est de chez nous ».

6 À écouter, à lire ou à observer ce que font certains de ceux qui feuillettent Pergaud, qui en parlent, qui sur lui écrivent, qui habitent ou ont habité des lieux pergaldiens dans le Doubs, qui participent de différentes façons à sa présence contemporaine - au-delà de sa seule lecture-, ou encore ceux qui tentent de le constituer en ressources intellectuelles, politiques et économiques, et par-là suivent Pergaud à la trace, ce ne sont pas à des "témoignages » que nous avons affaire - c'est-à-dire à des dispositifs 
oraux de restitution de circonstances passées et de transfert d'informations (Boutier, Fabiani \& Olivier de Sardan 2001) -, ou à de bonnes histoires - du pittoresque - mais à des propos réflexifs, à l'exercice d'une capacité critique et de mise en ordre d'un passé percolé par le présent, ainsi qu'à de multiples expériences sociales et pratiques qui permettent de le faire, soit une remémoration critique ${ }^{8}$. L'enquête, au-delà de sa dimension idiographique, a tenté de dégager les façons dont l'écrivain est aujourd'hui saisi par différents acteurs : enseignants, écoliers, chasseurs, habitants du "pays de Pergaud», parents de l'écrivain, admirateurs et servants, naturalistes... Ceux-ci parcourent les lieux de l'écrivain ou le célèbrent, parfois connaissent des épreuves existentielles pensées similaires.

7 C'est là, comme anthropologue, prendre la littérature par l'un de ses bouts : certaines des manifestations tangibles de l'attachement à un auteur et la construction des modalités de son existence - et leurs raisons. Mais c'est aussi laisser apparaître, dans et avec l'enquête, des points de cristallisation récurrents qui sont apparus dans la lecture des œuvres et les façons de les penser qui leur donne sens aux yeux des locuteurs: les relations entre humains et animaux et les qualités respectives qui s'y effectuent, les rapports sociaux et politiques que les humains entretiennent entre eux et les formes d'autorité qui s'y actualisent, les modalités et conditions d'une continuité culturelle, la place ou la posture d'où peut se dire une vérité sur un monde historique et social.

D'une certaine manière, ces points de cristallisation possèdent un degré de généralité et un pouvoir de généralisation; ils concernent la totalité des mondes historiques. L'enquête revint ainsi à réactiver une première fois Louis Pergaud, non plus saisi comme l'écrivain d'une charmante comédie enfantine portée au cinéma ${ }^{9}$, mais comme l'auteur d'une réflexion sur la possibilité de nos mondes et de nos existences. Cela nous a conduit à faire retour de ces points de vue - c'est-à-dire équipés des résultats de l'enquête - sur l'œuvre et à réexaminer, avec les outils de l'anthropologie et de la philosophie politique, des textes qui évidemment ont déjà subi d'autres traitements disciplinaires : il s'agissait de prendre Pergaud à rebours, et de ce fait l'aborder par un autre bout de la littérature.

Si Pergaud est cet écrivain, alors une seconde opération de réactivation est possible : le dégager du régime documentaire qui fait de lui l'auteur d'une œuvre limitée à un moment particulier ou à une région singulière; l'extraire d'un statut de descripteur à qui l'on peut faire parfois des procès en vérité, à partir même, et paradoxalement, des versions locales de la figure de l'écrivain. Délier Pergaud d'un régime documentaire de sa lecture, c'est non seulement redonner de l'importance à certains textes moins connus (Les Petits Gars des champs, La Vie des bêtes, Les Carnets de Jean-Baptiste Hislé...), qui ne sont ni nouvelle ni roman, mais aussi relire ses nouvelles et ses romans à la lumière des premiers, dans une sorte d'entrelacement. Ce qui était assigné à une volonté descriptive prend alors une autre couleur, et le lieu comme le moment des écrits de fiction, moins qu'objets de description, sont là où s'inscrit une pensée sur le monde et son cours. Il s'agit donc d'opérer un jeu de bascule qui autorise à lire l'œuvre en tant qu'elle engage, une fois débarrassée d'un carcan qui l'enferme dans une description de la "petite patrie ${ }^{10}$ », comme une réflexion plus générale. Il est alors possible de s'y appuyer pour penser ce que peuvent être notre présent et notre futur, bien loin donc d'une commémoration qui, de toute manière et dans sa nature même, est incompatible avec la réactivation d'une pensée (Badiou 2009 : 147). Les questions débattues par les enfants de LaGuerre des boutons, soudain, sont empreintes d'une certaine gravité et 
d'une portée qui nous affecte : qu'est-ce que l'égalité et comment peut-elle être mise en œuvre concrètement, ici et maintenant et non comme une promesse toujours trahie? Qu'est-ce que le pouvoir et de quelle manière peut-on s'en échapper ou le destituer? Comment se gouverne-t-on avec justice? Les animaux de Pergaud sont dotés de pensées et agissent en conséquence ; ils font société entre eux et des communautés sont établies et réglées avec les hommes. Pergaud nous convoque pour penser le monde dans un commun. L'écrivain peut alors entrer de plain-pied dans une confrontation avec d'autres qui ont pensé ces objets ${ }^{11}$.

Photographie de plateau du film La Guerre des boutons, réalisé par Yves Robert d'après le roman de Louis Pergaud, 1961.

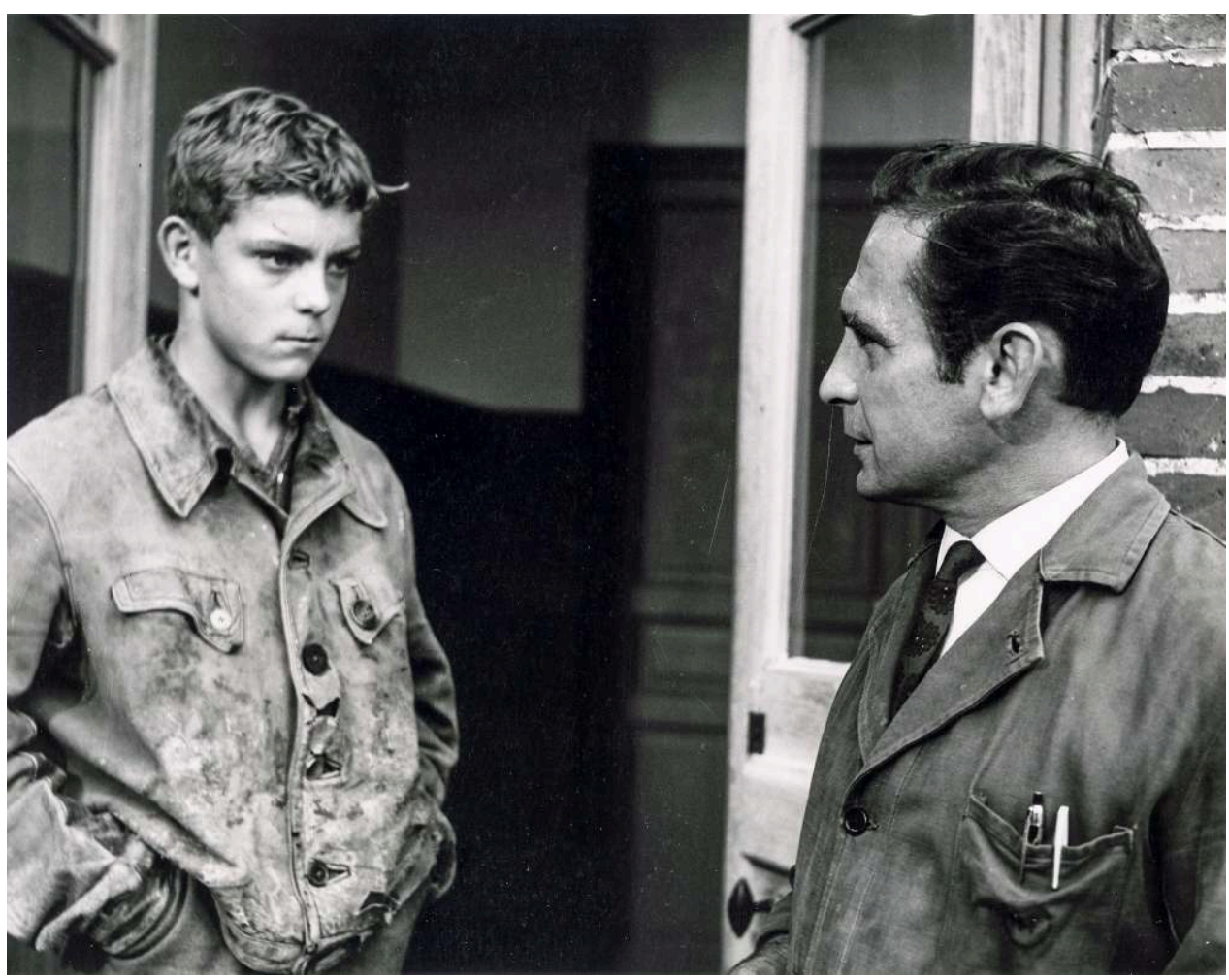

Si la relation de pouvoir consiste à faire faire, alors dans le roman La Guerre des boutons, ces derniers objets sont de remarquables instruments de pouvoir. Leur privation provoque l'humiliation de la bande adverse mais, plus que cela, opère une redistribution des capacités en «faisant faire » les parents de l'ennemi : en les faisant corriger leurs propres enfants. Constitués en « trésor », les mêmes boutons sont un dispositif de soustraction à l'exercice brutal d'une autorité parentale rendue sans objet. Celleci forme système avec les rapports entretenus par l'instituteur, le "père Simon », avec les enfants qui entendent s'en déprendre. Bienveillant dans le film d'Yves Robert, le personnage de l'instituteur est, dans le roman de Pergaud, considéré comme une « vieille andouille » et un « vieux salaud ». Il incarne aussi la promotion de catégories civiques abstraites contre l'égalité en actes de la bande d'enfants.

(c) Zazi films.

Mais aussi sur la question du lieu d'où peut se dire et s'énoncer la vérité d'un monde social : de Cacaine décrit dans les Rustiques, des Carnets de Jean-Baptiste Hislé, ou bien de La Crique dans La Guerre des boutons et dans Lebrac bûcheron... La réponse est nette et implacable: il s'agit des marges, des limites et des extériorités. Il s'agit de l'Autre comme position, ou bien de l'étrangeté dans une tension entre rupture et appartenance, ce que clairement écrit Pergaud dans Renaissance, poème tant de fois convoqué à demi-sens pour en faire un écrivain régional. Ce serait là une figure particulière de l'écrivain qui serait revendiquée et, d'un point de vue anthropologique, 
la limite sans cesse maintenue et réaffirmée qui serait le modèle d'interprétation du processus de création littéraire.

Eugène Atget, Chiffonniers, cité Valmy, Porte d'Asnières, 1913.

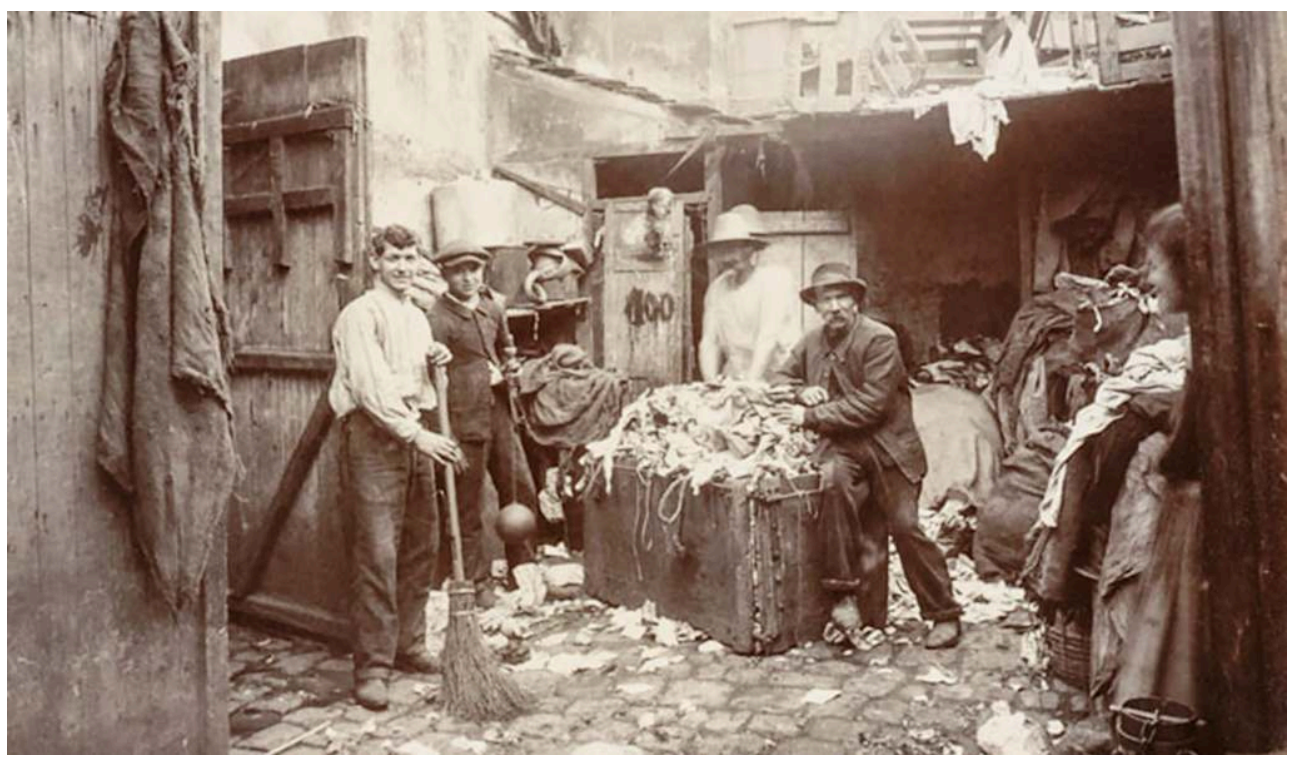

Échappant à l'historicisme et au positivisme, le « chiffonnier de l'histoire » décrit par Walter Benjamin - «collectionneur de haillons », à la marge lui-même - ramasse les déchets de l'histoire. À la recherche de correspondances et de constellations, pratiquant le montage historique, il crée l'histoire avec ses détritus même, tentant de saisir la « concrétude extrême d'une époque » à travers une situation de vie, un bâtiment... II s'agit de faire voler en éclat le temps continu de l'histoire et la cohérence rétrospective du passé, de repérer les chances et les tentatives de renverser l'ordre établi, d'ouvrir un temps des possibles.

(c) Musée Carnavalet / Paris Musées.

11 Reprenons le fil. Il s'est bien agi là de donner une position et une portée épistémologiques à l'expérience de lecteurs déterminés de Pergaud - rapidement dit leurs ascendants faisaient partie des mondes sociaux mis en narration par l'écrivain non pour les maintenir dans la localité d'une lecture et produire une analyse d'une réception particulière, mais bien pour les faire contribuer à une connaissance ou une théorie générale de Pergaud, à une "objectivité forte " pour reprendre les termes de Sandra Harding, l'une des théoriciennes de l'épistémologie située, qui soutient en particulier cette exigence : la prendre en compte, dès le départ de l'étude, des visions et des savoirs construits à partir de positions minoritaires (Harding 1991). Il ne s'agit pas d'essentialiser les «minoritaires », les positions ou les vies marginales, mais bien de les identifier à chaque fois en tant qu'ils peuvent être agents d'effets de savoir d'une portée générale, et contribuer à la production d'une critique des visions installées : ici, les lecteurs localisés de Pergaud le sont non en ce qu'ils auraient des souvenirs ou des anecdotes à raconter et dont la portée ne serait que documentaire, loin donc de l'autorité en matière de littérature, d'histoire régionale ou de l'exercice savant de la biographie à partir desquelles se sont construits les discours majoritaires sur Pergaud. Bref, il s'agit de désassujettir des savoirs de l'espace d'effectivité dans lesquels ils sont enfermés, de voir l'objet de connaissance comme un agent et non comme un écran ou une ressource (Haraway $2007: 130$ ), et de leur donner un avantage épistémologique. «C'est un principe d'expérience qui se laisse toujours vérifier que de dire que l'histoire est faite par les vainqueurs à court terme, que ceux-ci pourraient éventuellement la 
maintenir à moyen terme, mais qu'ils ne sauraient en aucun cas la dominer sur le long terme » (Koselleck 1997 : 238).

\section{Épistémologie politique de l'action patrimoniale}

12 S'agissant de l'exercice même de la fonction de conseiller pour l'ethnologie, la question des positions situées, loin du positivisme, est à travailler, à ouvrir en suivant deux traits structurels :

13 une inquiétude sur la continuité territoriale et temporelle d'une politique du patrimoine ethnologique au sein du ministère de la Culture, mesurable par exemple à la couverture du territoire national jamais réalisée et aujourd'hui en régression, ou aux questionnements récurrents sur "l'avenir de la Mission du patrimoine ethnologique ", soit la fragilité comme modalité d'existence ;

une certaine marginalité au sein des directions régionales des Affaires culturelles, qui, loin d'être assimilable à un repli dans une tour d'ivoire, tient par exemple à la répartition des budgets ou à la variabilité de l'inscription dans les organigrammes.

Cette position pourrait être lue sous le substantif «extraterritorialité » ou sous le qualificatif « intempestif » : ni porteur par exemple d'une vision patrimoniale classique (liée à la valeur historique) ou d'une vision documentaire de nouvelles catégories patrimoniales, ni convaincu de l'action culturelle au sens d'une maximisation de la diffusion de biens expertisés comme culturels ${ }^{12}$. Il en irait ainsi d'une extériorité aux points de vue dominants de l'administration de la culture, d'une hétérodoxie critique ${ }^{13}$ ou d'une hérésie ${ }^{14}$, entendus position et un trajet propres, à partir desquels il est possible de penser le patrimoine et l'action culturelle, une pensée encodée sous la formule " épistémologie politique de l'action patrimoniale ».

"Épistémologie », parce que l'activité patrimoniale est aussi activité d'élaboration et de production de savoirs. "Politique ", parce que l'activité épistémique consiste à doter les objets à connaître et les sujets capables de les connaître de qualités particulières, à qualifier les relations devant s'établir entre les deux. J'emprunte cette notion d'épistémologie politique à Bruno Latour (2008) lorsqu'il fait travailler les liens entre des conceptions qu'on se fait de la connaissance et celles qu'on peut se faire du politique. Politique doit s'entendre selon deux glissements. Le premier, au sens affirmé par Pierre Rosanvallon (2003 : 14) : «Se référer au politique et non à la politique, c'est parler du pouvoir et de la loi, de l'État et de la nation, de l'égalité et de la justice, de l'identité et de la différence, de la citoyenneté et de la civilité, bref de tout ce qui constitue une cité au-delà du champ immédiat de la compétition partisane pour l'exercice du pouvoir." L'exercice patrimonial a d'emblée à voir avec ces entités: le pouvoir, la loi, l'État, la nation, l'égalité... Et il convient que nous y soyons éthiquement, politiquement attentifs. Que produisons-nous comme spécialistes/ experts du patrimoine en termes de pouvoir? Quels rapports devons-nous entretenir avec les injonctions qui proviennent du pouvoir, par exemple avec les «valeurs de la république »? Le second glissement s'appuie sur la distinction et l'opposition faites entre " politique » et " police » par Jacques Rancière dans La Mésentente. Il vient cette fois à l'appui d'une micro-politique des pratiques patrimoniales. La notion de police cerne le principe du partage, la répartition des compétences et des rôles, des titres et des aptitudes, des capacités, autrement dit organise le rassemblement des êtres 
humains en une communauté hiérarchisée. La logique policière est une logique de distribution des places. La politique chez Rancière ne fait pas signe vers une lutte, un affrontement vers la conquête du pouvoir d'État ou dans l'État; elle renvoie au bouleversement des situations, des partages; elle repose sur la présupposition de l'égalité de tous et « commence avec l'existence de sujets qui ne sont "rien", qui sont en excès sur tout compte des parties de la population » (Rancière 1995 : 59). Elle est aux conditions de l'institution d'une part pour les sans-part (ibid. : 31).

17 "Action", enfin, puisqu'il faut bien une action pour faire patrimoine, dans une proximité avec l'action culturelle, lorsque celle-ci a pour ambition de donner sens à une situation $^{15}$, et parce que c'est dans l'action que se déploient places et capacités, égalité et résistances. C'est donc de ce point de vue situé, de cette position théorisée, de cette épistémologie politique de l'action patrimoniale que peut être lue, par exemple, l'histoire de la Mission du patrimoine ethnologique en tant qu'instrument de gouvernement des vivants selon un mode pastoral (Barbe 2013), où il ne s'agit pas de contraindre mais de faire faire au nom de la pratique d'une bonne science.

\section{Adieux à la « démocratie patrimoniale », vive la politique patrimoniale?}

18 À suivre ce fil d'une épistémologie politique du patrimoine, soit la distribution de qualités et la production de savoirs, à s'y appuyer dans la construction d'actions patrimoniales, nous avons encodé certains des dispositifs que nous avons construits sous le mot "démocratie ", désignant par-là, et en opposition à la démocratisation culturelle, une extension du - ou un écart par rapport au - cercle de l'expertise légitime œuvrant à l'attribution d'une valeur patrimoniale à tel ou tel bien, quelle que soit l'échelle de référence de la qualification, de l'exposition "participative» au jury citoyen comme dispositif de délibération, une sorte de pastorat démocratique (Brossat 2008).

19 Si l'encodage d'opérations culturelles sous le terme «démocratie patrimoniale » peut apparaître comme récent, d'un point de vue généalogique, les questions de "démocratie culturelle » ou de "participation » ont pourtant été mises à l'épreuve à différents moments et dans divers dispositifs et arènes, parfois liés : nouvelles formes d'action culturelle à la fin des années 1960 et au début des années 1970, écomuséologie, prospective en matière de développement culturel... Plus tard, sont venues les conventions européennes ou onusiennes, celle de Faro ou celle portant sur la sauvegarde du patrimoine culturel immatériel, publicisant la "participation » et la « démocratie».

20 Les gestes promus d'attribution de la valeur culturelle peuvent être politiquement caractérisés, que ce soit du point de vue d'un antagonisme recourant parfois au lexique des classes sociales ou, ensuite, par la promotion consensualiste de dispositifs techniques comme la participation - parfois par la suite fortement institutionnalisés, voire devenus objets de marché et fortement institutionnalisés (Blondiaux 2008) - en vue de la production de communautés patrimoniales, de la réfection du "lien social » ou d'une simple intensification de la fréquentation des lieux culturels.

21 Comment dans cette situation et face à cet héritage, transformer temporairement des systèmes d'expertise en sphères démocratiques, donner une visibilité à des contre- 
expertises ou favoriser des foyers d'expérience, faire un pas de côté par rapport ou contre l'autorité et l'académisme et tenter de faire surgir les rationalités d'un " gouvernement au patrimoine ». Faut-il alors parler de « démocratie patrimoniale»?

De ce point de vue, une expérience particulière conduite avec le parc naturel régional des Ballons des Vosges (PNRBV), largement décrite par ailleurs pour qu'il soit besoin d'y revenir ${ }^{16}$, s'est fondée sur la mise en place de jurys citoyens aux membres désignés par tirage au sort, appelés à délibérer pour attribuer une valeur patrimoniale à certains biens. Au-delà de certains dysfonctionnements dans l'accomplissement de ce processus - par exemple l'absence réelle des jurys dans une phase de mise en œuvre de leurs choix -, et malgré la dilatation provisoire produite de leur capacité d'agir pour certains des habitants du territoire du parc, la question du bien-fondé de l'expérience peut être posée, radicalement, c'est-à-dire à sa racine (Mondzain 2017). Le dispositif délibératif parce qu'il conduit d'abord à penser que le politique relève de la sphère de la parole, alors qu'il peut aussi s'effectuer et se réalise principalement dans l'affrontement des $\operatorname{corps}^{17}$, parce qu'il suppose que, dans la réalisation d'un consensus, par l'émission d'un avis unique réputé indivisible, se forme une unité, enfin parce qu'il postule l'existence d'un bien commun - autorise de multiples confusions : entre consensus et politique, alors que la conflictualité, y compris violente, est une modalité sine qua non du politique ; entre indétermination des fins du vivre ensemble qui, à suivre Claude Lefort (1994), caractérise la démocratie et les technologies consultatives; entre consensus et démocratie, alors que cette dernière ne peut se résumer à la production de l'Un; ou encore une fois entre consensus et politique, dans un monde social marqué par la division et les luttes qui s'y actualisent et la produisent tout à la fois ${ }^{18}$.

Pour cela, les dispositifs de délibérations sur la patrimonialité ne font en rien rupture et ne permettent que peu d'écart. Ils sont plutôt en adéquation avec les formes qu'ont prises les instances de gouvernement territorial, là ou ailleurs, où toute différence politique se trouve abolie au nom d'un principe supérieur commun: l'«intérêt du territoire ». Fabien Desage et David Guréanger, pointent et explorent la confiscation politique qu'opère par exemple le développement des intercommunalités, mode de "gouvernance » en vogue, dont l'un des principes de fonctionnement est le consensus inter partisans. «Loin d'un "parlement intercommunal", animé par des prises de parole contradictoires et les joutes oratoires, ces réunions sont des lieux à l'ambiance étonnamment policée et courtoise, où le fait de s'opposer apparaît souvent incongru " (Desage \& Guéranger $2011: 127$ ). En 2005, la Cour des comptes note dans un rapport sur l'intercommunalité que les décisions s'y «caractérisent souvent par la recherche systématique, non pas d'une adhésion majoritaire, mais d'un consensus le plus large possible ", tandis que l'Assemblée des communautés de communes de France montre dans une étude que s'y développe « un nouveau modèle d'élaboration de la prise de décision politique locale fondé sur le dialogue, la négociation et le consensus » (Desage \& Guéranger $2011: 126)$.

24 Autrement dit, si l'on considère que la démocratie repose sur le différend et l'écart (Grelet, Lèbre \& Wahnich 2009), l'installation et la mise au point de jurys citoyens n'en est guère. À l'objection recevable qui pourrait m'être faite de la production par cette expérience d'un espace ou d'une ouverture de la parole, peuvent être opposées les réalisations postérieures de deux projets menés en collaboration avec le PNRBV, qui viennent conforter l'absence d'écart dans laquelle doit, de son point de vue, se constituer la " parole des habitants », pourtant célébrée comme fondatrice. D’abord, en 
2014, un travail de recherche-action sur les manières dont les habitants de deux communes vosgiennes (Gérardmer, La Bresse) et d'une commune alsacienne (Ammerschwir) qualifiaient l'architecture issue de la reconstruction après la Seconde Guerre mondiale ${ }^{19}$. Quand bien même les qualifications dégagées devaient être intégrées à des politiques urbaines, il faut se rendre à l'évidence qu'elles se trouvaient poussées aux marges, comme un à-côté, tantôt comme une ressource documentaire visant à alimenter un "sentier de la mémoire ", tantôt pour penser les façons de convaincre les habitants, malgré ou contre ce qu'ils pensent, qu'il s'agit bien là de patrimoine - en quoi nous retrouvons la position pastorale. L'exercice revenait en définitive à confiner les sciences sociales - qui souhaitent par ailleurs s'extraire d'un rôle d'expertise ou de l'exercice d'une sociocratie positiviste, ou bien comme ici plutôt d'un simple dispositif de phonation (Latour 1999) -, à un rôle ancillaire. Le second exemple, plus récent, illustre la confusion entre politique et absence de dissensus, ou du moins de dissensus visible. En 2016-2017 une enquête en sciences sociales, en HauteSaône, plus précisément dans les Vosges saônoises, a entendu travailler sur les conceptions qu'ont des éleveurs de leur métier, de son présent, de son exercice et de ses conditions ${ }^{20}$. Les résultats devaient en être rendus publics lors d'une exposition dans un musée appartenant au conseil départemental de la Haute-Saône, jusqu'à ce que survienne une crise dont le projet d'affiche de l'exposition servit de prétexte. Pour ce support de communication, les chercheurs avaient choisi une photographie en relation avec l'un des éléments récurrents des propos des agriculteurs des Vosges saônoises, à savoir l'utilité de leur métier et de sa production. Sur la photographie, en noir et blanc, un petit camion-citerne déverse son chargement, du lait, dans le fossé et sur la route, tandis qu'un groupe de personnes discutent à l'avant. Bien qu'ancienne, l'image fut jugée trop conflictuelle ; elle n'aurait donné à voir ni " une bonne image du territoire » ou sa spécificité ${ }^{21}$, pas plus qu'elle n'aurait permis de le «faire avancer »-mais vers quoi ? À la manœuvre, le conseil départemental de la Haute-Saône, mais aussi le PNRBV- peut-être -, étrangement allié avec une chambre d'agriculture tenue par la FDSEA, parti prenant, sous couvert d'une posture apolitique, d'une politisation dénonçant le caractère « engagé » de l'exposition, et cherchant à l'entraver. 
Marc Paygnard, Camion déversant sa cargaison de lait, Haute-Saône, début des années 1970.

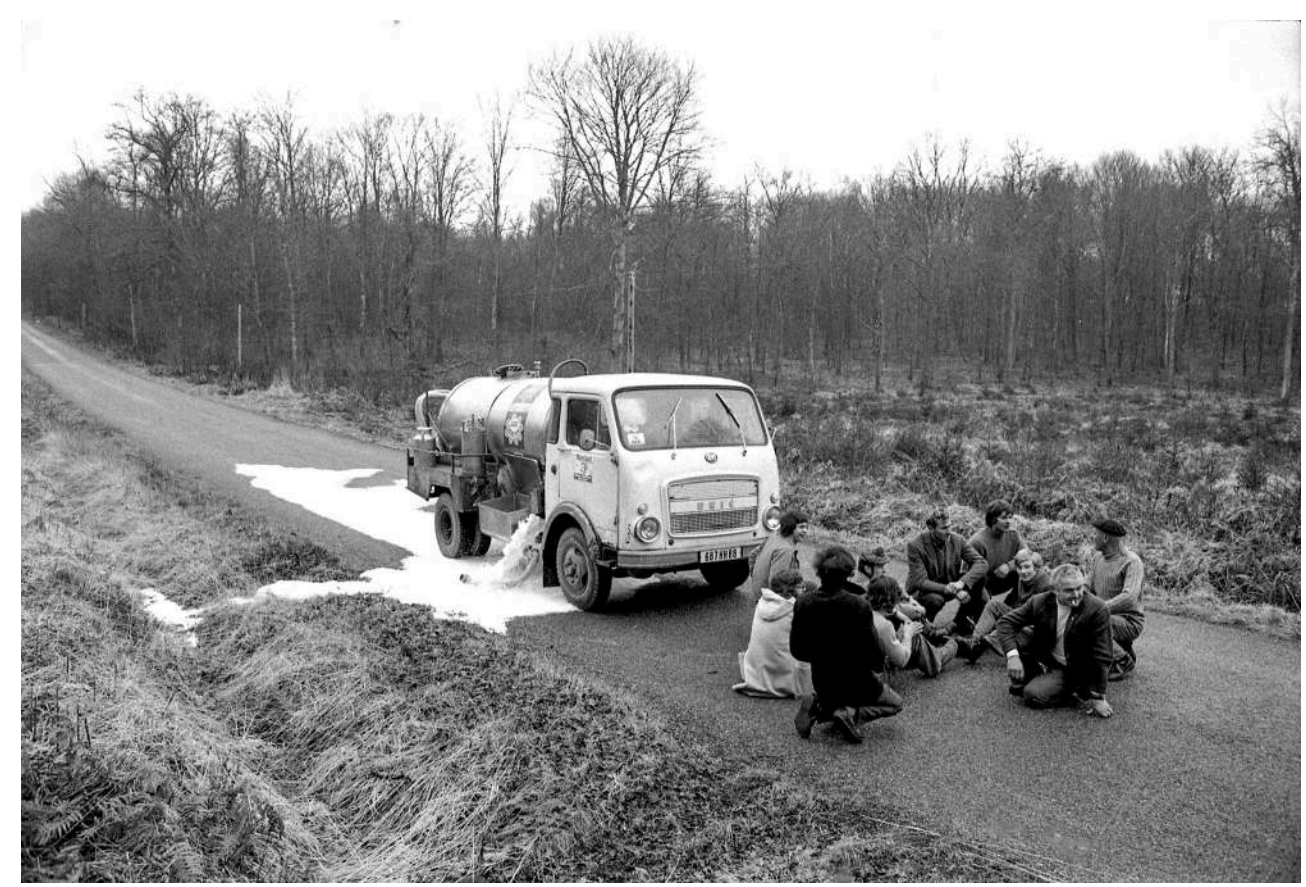

Destructions de bidons, vidage de camions citernes, déversement de sa propre production dans une fosse à lisier ou dans les champs devant les caméras, la « grève du lait » - et plus largement la destruction des productions - appartient au registre d'action des agriculteurs lorsqu'il s'agit, par exemple, de peser sur le prix des produits agricoles ou de lutter contre leur effondrement. À partir des années 1970, le processus de concentration des entreprises de transformation et de distribution laitières, la spécialisation des producteurs, puis l'abandon de la régulation du marché du lait par I'Union européenne au profit d'une concurrence promue comme le meilleur mode de régulation, ont accentué la pression sur les producteurs.

(c) Marc Paygnard.

D'un côté donc, la constitution d'un espace public de type habermassien, dont on connaît les limites, et un aplanissement des oppositions qui tous deux font peu démocratie; de l'autre, différents essais de constituer des contre-espaces, qui achoppent sur les représentants d'une démocratie représentative, dont la crise est largement reconnue. Reste à s'interroger sur la dimension «insurgente» d'une épistémologie politique du patrimoine.

\section{Retour}

Il faut voir dans les cas évoqués plus haut des symptômes, celui de la pensée d'une posture construite, à l'occasion de cas, dans une marge institutionnelle, selon ce qu'elle fait faire ou autorise ou selon ce qu'on peut y faire, et celui de la défectuosité d'un emplacement pour la faire vivre. La posture peut être lue comme plébéienne par sa dimension projective, par la puissance d'agir qu'elle propose, par sa revendication d'égalité. Elle appelle ce que Didier Éribon $(2001: 307,319)$ a nommé, dans son travail sur Jean Genet, une «morale du minoritaire » marquée par le provisoire et l'éphémère, par la discontinuité au sein d'un monde marqué par le continu, par une tension permanente entre intégration et écart. 
Benjamin Larderet, Chantier de percement du tunnel de la ligne TGV Lyon-Turin. Opposants No TAV s'éloignant du canon à eau de la police lors d'un rassemblement devant les grilles du chantier. Chiomonte, Val Susa, Italie, 2012.

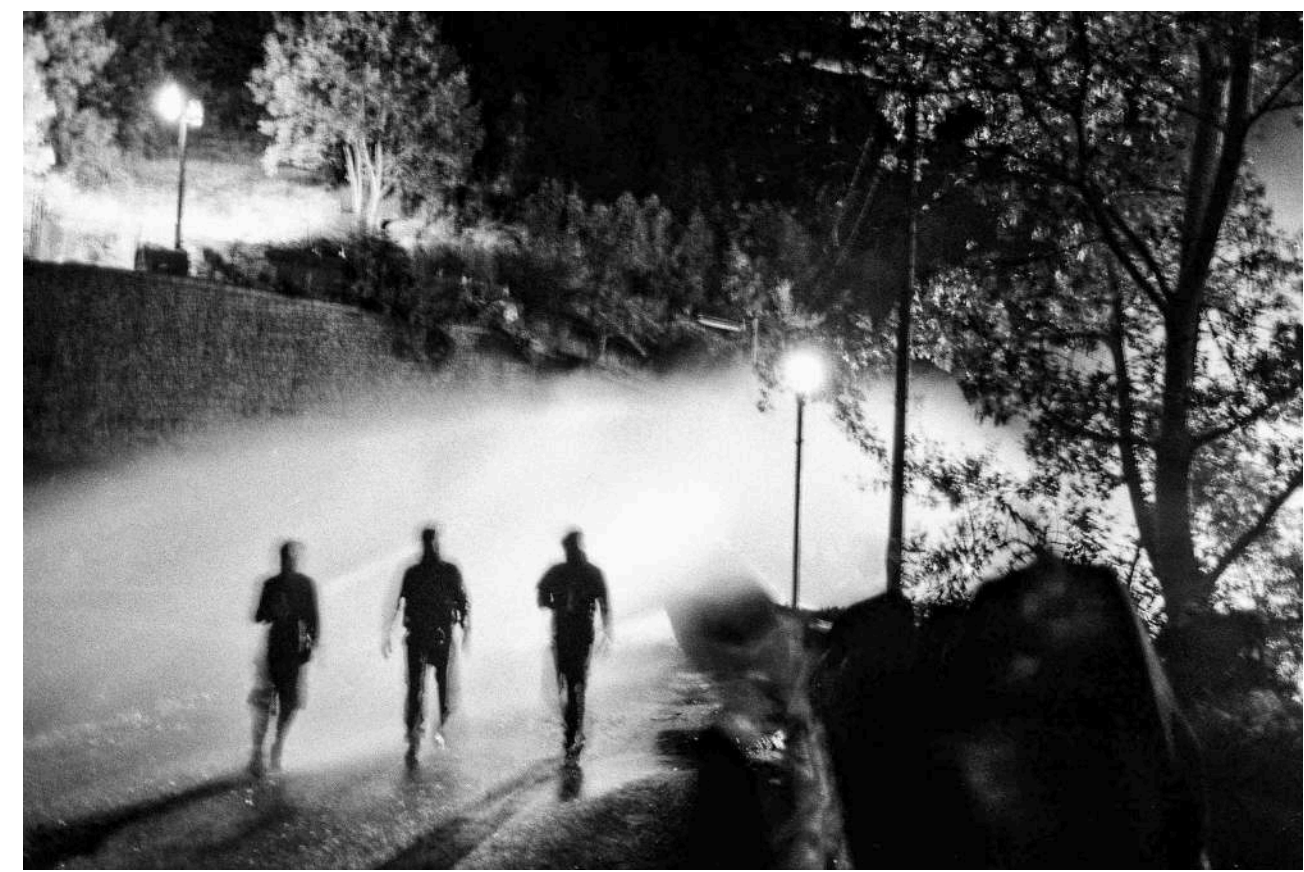

Dans la vallée alpine de Susa s'est construit un mouvement populaire d'opposition au projet de construction d'une nouvelle ligne ferroviaire à grande vitesse Lyon-Turin : le No TAV. De nombreuses manifestations sont organisées, des occupations de lieux décidés, une expertise populaire réglée, des sabotages de machines et attaques de chantier effectuées.

(c) Benjamin Larderet.

27 Une autre lecture appelle la défection et la déprise - au sens de : se déprendre de -, ou la destitution ${ }^{22}$, la " pratique du braconnage en bande » (Deleuze \& Parnet 1996 ; Barbe \& Sevin 2005) en ce qu'il est jeu avec la limite, la "guérilla » diraient certains, en ce qu'elle accroche l'ennemi et décroche de sa position, et en ce qu'elle est rappel sans fin $^{23}$ de la possibilité d'autres mondes futurs et présents. Dans la posture ici objectivée, c'est d'une mise en présence stratégique du passé des politiques patrimoniales dont nous avons besoin. Il s'agirait dès lors :

de retravailler la question du " populaire », devenue proliférante, non dans le sens d'une strate sociale spécifique ou d'un moment historique - hors donc des publics, d'une sociologie ou des "arts et traditions populaires»-, mais d'un faire-action patrimonial en tant qu'il constitue du peuple, qu'il porte une revendication en égalité, et manifeste une exclusion ne réclamant aucune inclusion ;

d'analyser l'expérience des vaincus en tant qu'elle vient s'affronter et mettre en tension les modes de pensée et de faire qui justifient la domination ou l'oubli des expériences émancipatrices et des résistances, et de façon conséquente déconstruire les mythes de ceux qui restent dans l'histoire ${ }^{24}$;

de prêter attention à ce que pourrait être un patrimoine de la rupture c'est-à-dire à des formes de présence d'expériences de résistance à l'ordre inacceptable du monde tel qu'il s'annonce ou qu'il est déjà là, ou bien celles de moments de bascule dans un nouveau monde socio-historique ; 

se peut, à ces expériences ${ }^{25}$.

Gustave Courbet, Révolutionnaire sur une barricade, projet de frontispice pour Le Salut public, 1848.

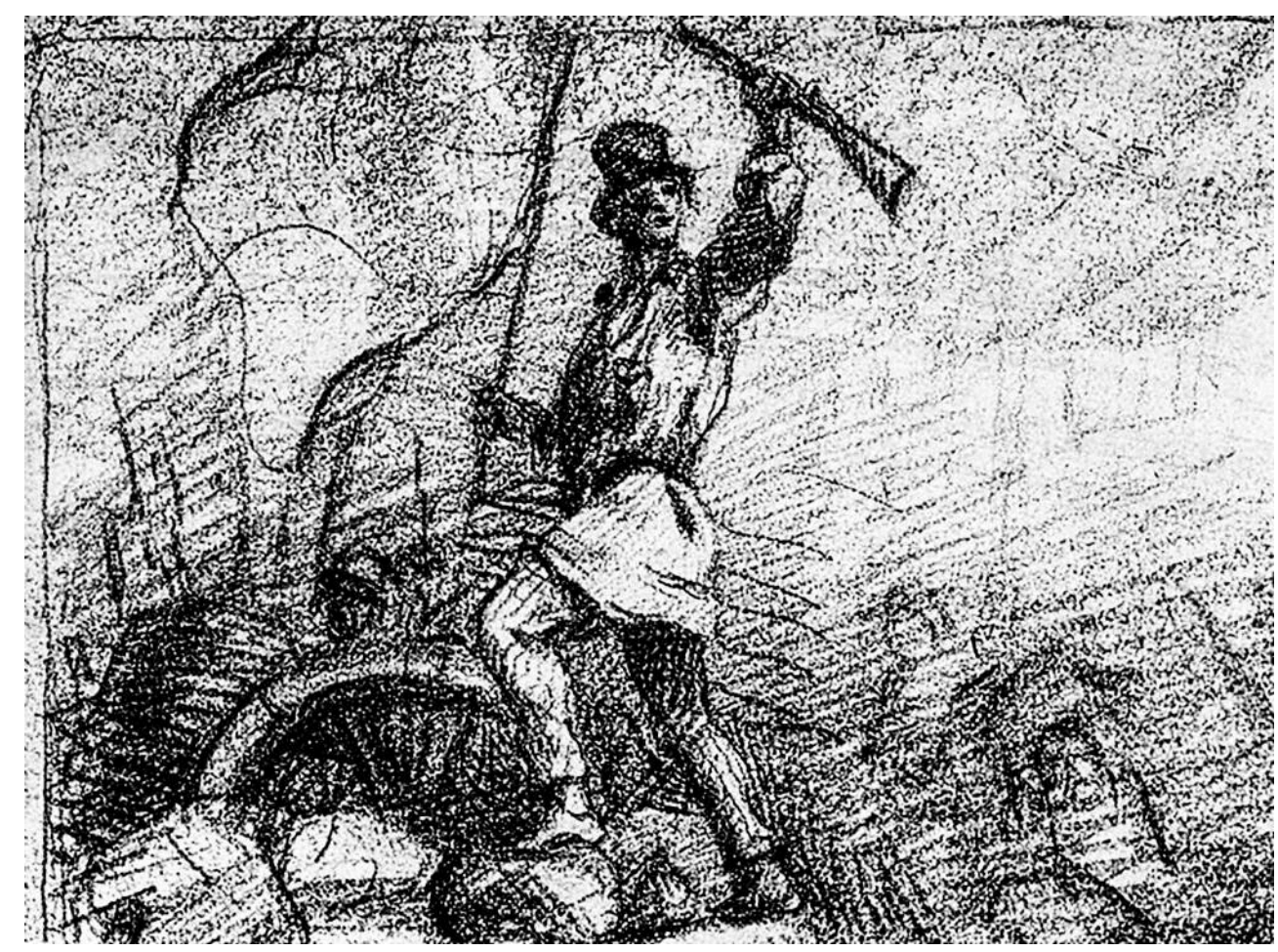

Durant les journées de 1848, on dit Courbet passif. II accepte pourtant de dessiner un frontispice pour le deuxième et dernier numéro de Salut public, journal dont le nom trouvé par Baudelaire fait écho au Comité de salut public constitué en 1793 pour protéger la révolution. Alors que Delacroix place le public face à la Liberté guidant le peuple, Courbet l'installe à l'intérieur de la barricade. C'est nous, regardeurs, que l'homme en blouse et haut de forme, brandissant un fusil tenu au bout de son bras levé, entend entraîner. Autrement dit, nous voilà appelés à faire de la barricade notre affaire. Plus tard, le 14 août 1866, répondant à un rédacteur du Monde illustré qui lui reproche sa « mauvaise éducation », Gustave Courbet revendique son « origine plébéienne ».

(c) Musée Carnavalet / Paris Musées. 
Le vif plutôt que le mort, Lip ça peut toujours servir...

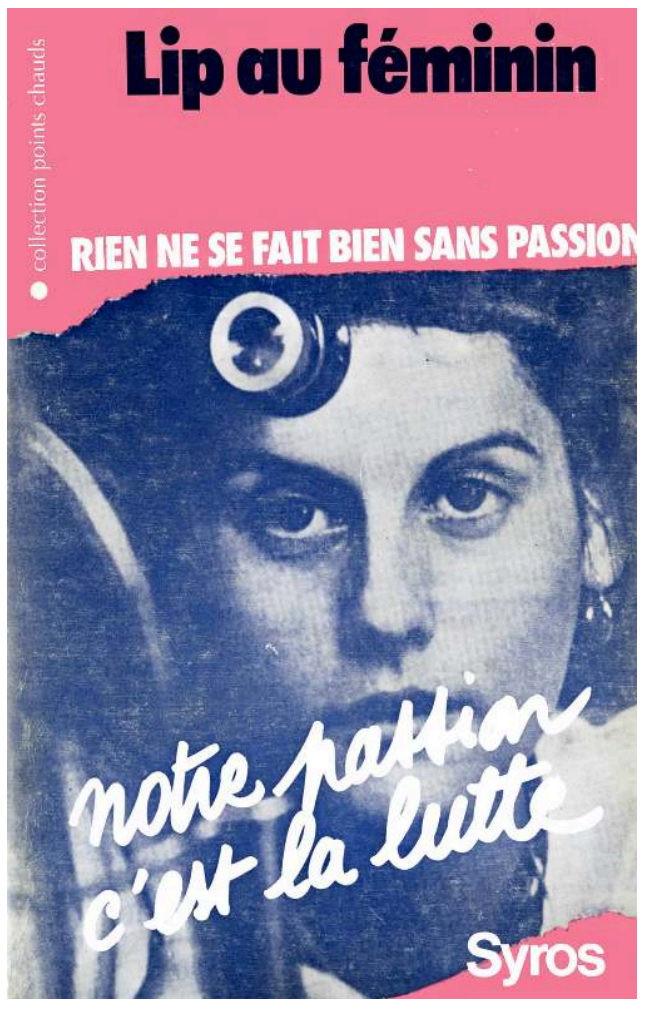

Fruit d'une réflexion collective, la brochure Lip au féminin paraît en février 1975, tirée à 9000 exemplaires. Traduite en de nombreuses langues étrangères et rapidement épuisée, elle est republiée en 1977 sous forme de livre. Expérience de subjectivation ancrée dans une situation, les femmes de Lip y parlent de leur vie au travail, à la maison... Le livre s'attache à faire entendre des voix, de faire valoir des expériences. Ces témoignages ont plus de quarante ans, pour autant la portée générale que ces femmes entendent leur donner, comme la soif d'égalité et de démocratie qui les anime, vient tout à la fois troubler et alimenter le présent. En ce sens, Lip n'est pas derrière nous, mais nous sommes bien devant Lip.

(c) Éditions Syros.

\section{BIBLIOGRAPHIE}

AILHAUd Flavie \& BARBE Noël (dir.), 2017, Pergaud... l'Autre, Besançon, Éditions du Sekoya, coll. « Les Cahiers de l'ethnopôle ».

BADIou Alain, 2009, L'Hypothèse communiste, Paris, Lignes, coll. « Circonstances ».

BADIOU Alain, 2016, Notre mal vient de plus loin. Penser les tueries du 13 novembre, Paris, Fayard, coll. « Ouvertures ».

BARBE Noël \& DUMAIN Aurélie, 2018, « L'architecture de la reconstruction. Une épreuve de patrimonialité et de souveraineté ", In Situ. Revue des patrimoines, $\mathrm{n}^{\circ} 37$, « Jardins collectifs : de l'abbé Lemire aux jardins d'insertion. Typologies, expériences, enjeux de conservation » [en ligne], https://journals.openedition.org/insitu/19834 [lien valide en octobre 2019]. 
BARBE Noël \& SEVIN Jean-Christophe, 2005, « Rencontre avec un braconnier de l'archive », Sociétés \& Représentations, $\mathrm{n}^{\circ}$ 19, vol. 1, « Lieux d'archives », p. 65-75.

BARBE Noël \& SEVIN Jean-Christophe, 2015, Le Retour des paysans de Flagey. Courbet à Flagey, politique et esthétique des Hauts-lieux, Besançon, Les éditions du Sekoya, coll. « Les Cahiers de l'Ethnopôle ».

BARBE Noël 2015, «Les revenances de Louis : domestiquer Pasteur ou être domestiqué », in BARBE Noël, RAICHVARG Daniel (dir.), Les Vies de la pasteurisation. Récits, savoirs, actions (1865-201), Dijon, Éditions universitaires de Dijon, coll. « Art, archéologie \& patrimoine », p. 245-254.

BARBE Noël, 2013, « Isac Chiva, ethnologie et politique patrimoniale », Terrain, $\mathrm{n}^{\circ} 60$, «L'imaginaire écologique », p. 148-163. Disponible en ligne, http://journals.openedition.org/ terrain/15127 [lien valide en mars 2018].

BARBE Noël, ChAULIAC Marina \& TORNATORE Jean-Louis, 2015, « Intangible cultural heritage exposed to public deliberation: a participatory experience in a Regional Nature Park », in ADELL Nicolas, BENDIX Regina F., BORTOLOTTO Chiara, TAUSCHEK Markus (dir.), Between imagined communities and communities of practice, Göttingen, Universitätsverlag Göttingen, « Göttingen Studies in Cultural Properties ", p. 201-2108.

BENSAïD Daniel, 2006, «Clercs et chiens de garde », Contretemps. Revue de critique communiste, $\mathrm{n}^{\circ} 15$, «Clercs et chiens de garde », p. 25-35.

BLONDIAUX Loïc, 2008, « Démocratie délibérative vs. démocratie agonistique ? Le statut du conflit dans les théories et les pratiques de participation contemporaines », Raisons politiques, $\mathrm{n}^{\circ} 30$, vol. 2, « Les victimes écrivent leur histire », p. 131-147. Disponible en ligne, https:// www.cairn.info/revue-raisons-politiques-2008-2-page-131.htm [lien valide en octobre 2019].

BOUTIER Jean, FABIANI Jean-Louis \& OLIVIER DE SARDAN Jean-Pierre, 2001, Corpus, sources, archives [en ligne], Tunis, Institut de recherche sur le Maghreb contemporain (IRMC), coll. «Études et travaux de l'IRMC », http://books.openedition.org/irmc/110?lang=fr [lien valide en mars 2018].

BREAUGH Martin, 2007, L'Expérience plébéienne. Une histoire discontinue de la liberté politique, Paris, Payot \& Rivages, coll. «Critique de la politique ».

BRosSAT Alain, 2003, Le Serviteur et son maître. Essai sur le sentiment plébéien, Paris, Léo Scheer, coll. « Lignes ».

BRossAT Alain, 2006, La Résistance infinie, Paris, Lignes, coll. « Lignes essais ».

BROSSAT Alain, 2008, « Les perversions du pastorat démocratique », Raison présente, $\mathrm{n}^{\circ}$ 166, p. 31-40.

BRossat Alain, 2013, Les serviteurs sont fatigués (les maîtres aussi), Paris, L'Harmattan, coll. « Quelle drôle d'époque !».

BRossat Alain, 2016, « Pour une politique plébéienne », Ici et Ailleurs. Association pour une philosophie nomade [blog], https://ici-et-ailleurs.org/voyons-ou-la-philo-mene/article/pour-unepolitique-plebeienne [lien valide en juin 2018].

BURAWoy Michael, 2003, «L'étude de cas élargie. Une approche réflexive, historique et comparée de l'enquête de terrain », in CEFAÏ Daniel (dir.), L'Enquête de terrain, Paris, La Découverte, coll. « Recherches », série « Bibliothèque du MAUSS », p. 425-464.

Chanial Philippe, 2011, La Sociologie comme philosophie politique, et réciproquement, Paris, La Découverte, coll. « Bibliothèque du MAUSS ». 
COMITÉ INVISIBLE, 2017, Maintenant, Paris, La Fabrique éditions.

DEBORD Guy, 2006 [1974], « Rapport sur la construction des situations », in CEuvres, Paris, Gallimard, coll. « Quarto», p. 308-329.

DELEUZE Gilles \& PARNET CLAIRE, 1996, Dialogues, Paris, Flammarion, coll. « Champs ».

DESAGE Fabien \& GUÉRANGER David, 2011, La Politique confisquée. Sociologie des réformes et des institutions intercommunales, Bellecombe-en-Bauges, Éditions du Croquant, coll. « Savoir-agir ».

DONZELOT Jacques, 1994, L'Invention du social. Essai sur le déclin des passions politiques, Paris, Éditions du Seuil, coll. « Points », série « Essais ».

DoRlin Elsa, 2017, Se défendre. Une philosophie de la violence, Paris, Zones.

ÉRIBON Didier, 2001, Une morale du minoritaire. Variations sur un thème de Jean Genet, Paris, Fayard, coll. « Histoire de la pensée ».

Foucault Michel, 1992, Histoire de la sexualité, tome 2, L'Usage des plaisirs, Paris, Gallimard, coll. «Bibliothèque des histoires ».

FOUCAULT Michel, 2001 [1974], « La vérité et les formes juridiques », in FOUCAULT Michel, Dits

et Écrits, tome 1, 1954-1975, Paris, Gallimard, coll. « Quarto », p. 1406-1514.

FoucAult Michel, 2012, Mal faire, dire vrai. Fonction de l'aveu en justice. Cours de Louvain, 1981, Louvain, Presses universitaires de Louvain.

GRELET Stani, LÈBRE Jérôme \& WAHNICH Sophie, 2009, « Insistances démocratiques. Entretien avec Miguel Abensour, Jean-Luc Nancy \& Jacques Rancière », Vacarmes, n 48, vol. 3, p. 8-717, disponible en ligne https://www.cairn.info/revue-vacarme-2009-3-page-8.htm [lien valide en juin 2018].

HABERMAS Jürgen, 2005, Théorie et Pratique, Paris, Payot, coll. « Critique de la politique ».

HARAWAY Donna, 2007, Manifeste cyborg et autres essais. Sciences, fictions, féminismes, Paris, Exils, coll. « Essais ».

HARDING Sandra G., 1991, Whose science? Whose's knowledge? Thinking from women's lives, Ithaca, Cornell University Press.

INGoLD Tim, 2017, Faire. Anthropologie, archéologie, art et architecture, Bellevaux, Éditions Dehors. JEANSON Francis, 1973, L'Action culturelle dans la cité, Paris, Éditions du Seuil.

KALINOWSKI Isabelle, 2005, « Leçons wébériennes sur la science et la propagande », in WEBER Max, La Science, profession et vocation, Marseille, Agone, coll. « Banc d'essais », p. 61-273.

KOSELLECK Reinhardt, 1997, L'Expérience de l'histoire, Paris, Gallimard / Éditions du Seuil, coll. « Hautes études ».

LATOUR Bruno, 1999, Politiques de la nature. Comment faire entrer les sciences en démocratie, Paris, La Découverte, coll. « Armillaire ».

LATOuR Bruno, 2008, «Pour un dialogue entre science politique et science studies ", Revue française de science politique, $\mathrm{n}^{\circ} 4$, vol. 58, p. 657-678.

LEFFEBVRE Henri, 1989, La Somme et le Reste, Paris, Méridiens Klincksieck, coll. « Analyse institutionnelle ».

LEFORT Claude, 1994, L'Invention démocratique. Les limites de la domination totalitaire, Paris, Fayard. 
LöWY Michael, 1990, « Karl Mannheim. Intellectuel sans attaches », Critique, n 517-518.

MONDZAIN Marie-José, 2017, Confiscation : des mots, des images et du temps, Paris, Éditions Les liens qui libèrent.

Mosès Stéphane, 2006, L'Ange de l'Histoire. Rosenzweig, Benjamin, Scholem, Paris, Gallimard, coll. « Folio », série « Essais ».

NAZE Alain, 2013 (4 novembre), « Pour une émancipation plébéienne », Ici et Ailleurs. Association pour une philosophie nomade [en ligne], https://ici-et-ailleurs.org/voyons-ou-la-philo-mene/ article/pour-une-emancipation (lien valide en novembre 2018).

RANCIÈre Jacques, 1995, La Mésentente. Politique et philosophie, Paris, Galilée, coll. « La philosophie en effet ».

rosanVallon Pierre, 2003, Pour une histoire conceptuelle du politique, Paris, Éditions du Seuil.

THOMPSON Edward P., 2015, Les Usages de la coutume. Traditions et résistances populaires en Angleterre, XVII ${ }^{e}$-XIX ${ }^{e}$ siècle, Paris, Éditions de l'EHESS / Gallimard / Éditions du Seuil, coll. « Hautes études».

WEBER Max, 1992, Essais sur la théorie de la science, Paris, Presses Pocket, coll. « Agora ».

yovel Yirmiyahu, 1991, Spinoza et autres hérétiques, Paris, Éditions du Seuil, coll. « Libre examen. Histoire de la pensée ».

zINN Howard, 2002, Une histoire populaire des États-Unis. De 1492 à nos jours, Marseille, Agone, coll. « Des Amériques».

zINN Howard, 2013, L'Impossible neutralité. Autobiographie d'un historien et militant, Marseille, Agone, coll. « Éléments ».

\section{NOTES}

1. Liée donc historiquement à la catégorie de patrimoine ethnologique et à la mission ministérielle du même nom.

2. Au sens où l'entend Daniel Bensaïd (2006:26) : «Intellectuel engagé ? Intellectuel guetteur et sentinelle? C'est toujours présupposer l'intellectuel libre de choisir sa cause en toute conscience ; comme s'il n'était pas lui aussi « embarqué » à son insu, sa part de liberté consistant à penser, pour mieux s'en émanciper, les déterminations biographiques, sociales, institutionnelles de cet embarquement. »

3. Si j'emprunte ces termes à Jacques Donzelot (2014), je n'en suis pas moins interrogatif sur l'usage qu'il en fait pour revenir sur son parcours.

4. Sur cette question voir Alain Brossat (2003, 2008, 2013, 2016), Martin Breaugh (2007) et Alain Naze (2013)

5. Pour une discussion de la science positive et ses conséquences épistémologiques, voir par exemple Michael Burawoy (2003) et Tim Ingold (2013:27-31).

6. Sur la Ferme Courbet, voir Noël Barbe et Christophe Sevin (2015). L'exposition Pergaud... l'Autre a été organisée, dans le cadre de l'ethnopôle Pays de Courbet, pays d'artiste, à la Ferme de Courbet à Flagey, du 18 décembre 2015 au 8 mai 2016 (Ailhaud \& Barbe 2017).

7. Il faut ici souligner que ladite réplique n'est pas de la plume de Pergaud mais qu'elle apparaît dans l'adaptation du roman pour le film éponyme d'Yves Robert sorti en 1962. 
8. Au sens de Walter Benjamin donc un acte de la conscience plus que le surgissement du souvenir (Mosès 2006). Cela suppose une politique non documentaire de l'entretien, le concevant comme une épreuve dans laquelle s'opèrent des opérations de qualification.

9. Il faudrait plutôt dire un roman transformé en charmante comédie enfantine par le cinéma.

10. Ce thème, bien identifié et documenté pour le XIX et le début du $\mathrm{XX}^{\mathrm{e}}$ siècle, continue à courir aujourd'hui.

11. Voir Flavie Ailhaud et Noël Barbe (2017).

12. Une variante de la théorie du ruissellement en somme.

13. Voir Michael Löwy (1990).

14. Si l'on entend par là le sens que lui donne l'historien de la philosophie Yirmiyahu Yovel (1991), soit être le produit d'une réflexion d'une époque et, en même temps, en rupture par rapport à la réflexion de cette époque. Ici, être le produit d'une institution et en son sein, en même temps qu'en rupture avec elle.

15. Voir à ce propos Francis Jeanson (1973) et le moment, au sens de Worms, des années 1970 en matière d'action culturelle. On pourrait aussi se référer à Guy Debord (2006) ou à la théorie des moments de Henri Lefebvre (1989 : 233-250).

16. Par exemple Barbe, Chauliac \& Tornatore (2015).

17. Ce que l'État s'est chargé de nous rappeler, si besoin était, tant à Sivens qu'à Bure, à NotreDame-des-Landes ou lors des manifestations contre la loi El Khomri. Sur la violence comme moyen de faire l'histoire, du côté des dominés, voir Elsa Dorlin (2017).

18. On pourra aussi se reporter à Badiou (2016).

19. Voir Noël Barbe et Aurélie Dumain (à paraître).

20. Enquête conduite avec Camille Cusnier et Aurélie Dumain.

21. On ne voit pas de quelle façon les Vosges du Sud pourraient échapper aux politiques agricoles, à la puissance des industries agroalimentaire, au capitalisme, à la crise climatique...

22. Au sens développé par le Comité invisible (2017).

23. Au sens employé par Alain Brossat (2006).

24. Voir par exemple Noël Barbe (2015).

25. Leurs lecteurs auront reconnu ici l'influence tant d'Edward Zinn (2002, 2013) que de Edward P. Thompson (2015).

\section{RÉSUMÉS}

Ce texte s'origine dans un débat avorté et qui aurait pu porter sur l'intérêt de faire une histoire positiviste de l'un des modes de présence d'une discipline académique au sein de l'appareil d'État français, la Mission du patrimoine ethnologique.

À rebours du dualisme méthodologique promu par le positivisme, il s'agira ici de faire travailler la question d'un patrimoine plébéien et, pour cela, de déployer en regard certains des écarts et des oppositions mis à l'épreuve d'une pratique située et incarnée soit depuis l'exercice d'un travail tout à la fois de recherche et d'action, en tant que ce lien peut être négocié dans la situation de conseiller pour l'ethnologie dans une direction régionale des Affaires culturelles (Drac), par conséquent dans une position embarquée mettant à l'épreuve une épistémologie politique de l'action patrimoniale et une politique de gestes, mais doté aussi d'une boussole politique réglée au fil du temps de l'exercice et des projets, une boussole qui n'était pas donnée 
d'avance et qui se déploie, tant biographiquement qu'intellectuellement, entre « depuis le bas » et « depuis la marge ».

The origins of this text lie in an aborted debate which could have shown the interest of writing a positivist history of one of the modes of presence of an academic discipline within the state apparatus, the Ethnological Heritage Mission at the French Ministry of Culture. But unlike the methodological dualism that positivism promotes this text will attempt to work on the question of a plebeian heritage. In order to do this, it will confront certain deviations and oppositions tested by a situated and incarnated practice, that is to say work that was both research and action, in so far as this link can be negotiated in the situation of an ethnological advisor in a Regional Directorate of Cultural Affairs (Drac), consequently in an embedded position taxing a political epistemology of heritage action and a policy of gestures, but equipped too with a political compass ordered over time in exercise and projects, but a compass not given at the outset and which deploys itself, biographically and intellectually, between "from the bottom" and "from the margin".

INDEX

Mots-clés : action patrimoniale, plèbe, populaire, patrimonialisation, épistémologie politique

Keywords : heritage action, plebeian, popular, heritagisation, political epistemology

\section{AUTEUR}

\section{NOËL BARBE}

Anthropologue associé à l'Institut interdisciplinaire d'anthropologie du contemporain (IIAC, UMR CNRS 8177) et conseiller pour l'ethnologie et les sciences sociales à la Drac BourgogneFranche-Comté 